\section{Efetividade dos Centros de Atenção Psicossocial no cuidado a portadores de sofrimento psíquico em cidade de porte médio do Sul do Brasil: uma análise estratificada}

\author{
The effectiveness of Psychosocial Care Centers for \\ the mentally ill in a medium-sized city in southern \\ Brazil: a stratified analysis
}

\author{
1 Programa de Pós-graduação \\ em Saúde e Comportamento, \\ Universidade Católica de \\ Pelotas, Pelotas, Brasil. \\ 2 Programa de Pós-graduação \\ em Epidemiologia, \\ Universidade Federal de \\ Pelotas, Pelotas, Brasil. \\ 3 Departamento de Medicina \\ Social, Universidade Federal \\ de Pelotas, Pelotas, Brasil. \\ 4 Faculdade de Enfermagem \\ e Obstetrícia, Universidade \\ Federal de Pelotas, Pelotas, \\ Brasil. \\ Correspondência \\ E. Tomasi \\ Programa de Pós-graduação \\ em Saúde e Comportamento \\ Universidade Católica de \\ Pelotas. \\ Rua Almirante Barroso 1202 \\ sala 109-G, Pelotas, RS \\ 96010-280, Brasil. \\ tomasiet@uol.com.br
}

\section{Abstract}

The objective was to analyze the effectiveness of Psychosocial Care Centers (PCCs) for persons with mental disorders in Pelotas, Rio Grande do Sul State, Brazil. This was a prospective cohort study with users of the centers, based on two home visits. Three treatment exposure variables and two health status variables were compared and stratified according to treatment modality and time attending the health service. We interviewed 1,013 patients on the first visit and 875 on the second. The most frequent diagnoses were mood disorder (39\%), schizophrenia (24\%), and neuroses (13\%). More than $50 \%$ were receiving intensive or semiintensive care, and nearly $60 \%$ had attended the center for at least three years. There was a significant reduction in crisis reports $(p=0.000)$ and fewer hospitalizations among those in intensive treatment $(p=0.002)$ and with longer time attending the center $(p=0.003)$. Among non-intensive patients, there was a decrease in the use of medication ( $p=0.032)$ and increased participation in groups $(p=0.000)$. The Psychosocial Care Centers can be considered effective for persons with mental disorders.

Mental Health Services; Health Services Evaluation; Psychological Stress
Elaine Tomasi 1

Luiz Augusto Facchini 2

Roberto Xavier Piccini 3

Elaine Thumé 4

Ricardo Azevedo da Silva 1

Helen Gonçalves 2

Suele Manjourany Silva ${ }^{2}$

\section{Introdução}

O adoecimento psíquico é um problema de saúde pública que afeta indivíduos em todo o mundo, independente do desenvolvimento econômico do país. Estudo conduzido pela WHO World Mental Health Survey Consortium ${ }^{1}$ ouviu mais de 60 mil adultos em 14 países e registrou que a presença de algum transtorno psíquico - avaliado pelo DSM-IV (Diagnostic and Statistical Manual of Mental Disorders - Fourth Edition) - variou de $4 \%$ em Xangai, na China, a 26\% nos Estados Unidos. Apesar das limitações metodológicas para a quantificação da morbidade psiquiátrica em amostras populacionais, dadas a diversidade de instrumentos, contextos locais e patologias, é possível obter informações confiáveis da magnitude desses problemas.

No Brasil 2, estudo realizado com adultos aferindo prevalências de pelo menos um diagnóstico do DSM-III (Diagnostic and Statistical Manual of Mental Disorders - Third Edition), encontrou $61 \%$ em Brasília, 35\% em São Paulo e 52\% em Porto Alegre (Rio Grande do Sul). Em Pelotas (Rio Grande do Sul), a prevalência de distúrbios psiquiátricos menores em adultos, medida pelo SRQ-20 (Self Report Questionnaire), foi de 28,5\% no ano de 2000 3. Estimativas de esquizofrenia na população em geral situam-se em torno de $1 \%{ }^{4}$ e os transtornos bipolares atingem de $3 \%$ a $6 \%$ da população ${ }^{5}$. Há relatos de prevalência de depressão de cerca de 10\% em Porto Alegre, 13\% 
nos Estados Unidos e 10\% na Grã-Bretanha 6. Os transtornos associados à dependência de álcool e drogas atingem $6 \% 7$ a $14 \% 8$ e $12 \%$, respectivamente, e os transtornos de ansiedade generalizada são encontrados em $11 \%$ de idosos 9 .

A assistência aos portadores de sofrimento psíquico no Brasil vem se transformando nas últimas décadas, influenciada por experiências internacionais que propuseram novos modelos e práticas de transformação institucional com intuito da promoção da saúde mental fora do âmbito manicomial 10,11,12. Entre as estratégias assistenciais, foi proposta a criação de Centros de Atenção Psicossocial (CAPS) com o objetivo de oferecer atendimento à população moradora na área de abrangência em um modelo que prioriza a reabilitação e a reintegração psicossocial do indivíduo adoecido mentalmente, mediante acesso ao trabalho, lazer, exercício dos direitos civis e fortalecimento dos laços familiares e comunitários 13 .

Borges \& Baptista 14 traçam um panorama detalhado e preciso da evolução das políticas em saúde mental de 1991 a 2004 em nosso país, identificando para o último período analisado - 2002 a 2004 - um momento de expansão do modelo assistencial, com destaque para os CAPS e as residências terapêuticas. Na consolidação da reforma psiquiátrica no Brasil, observa-se a ampliação consistente no número de CAPS, principal serviço alternativo deste modelo. O total de unidades cresceu de 154 em 1996 para 295 em 2001, para 546 em 2004 e para 1.153 em 2007 15. Paralelamente à expansão, faz-se necessária a avaliação dos serviços substitutivos em saúde mental, mais especificamente para analisar o desempenho 16 de tais políticas e serviços, suas possibilidades e limitações.

Em Pelotas, há mais de duas décadas, os trabalhadores da saúde pública do município e região se unem em um processo de luta em favor de um novo modelo de atenção em saúde mental, com desafios assistenciais de grande magnitude e conquistas políticas importantes 17. Em 2002, acompanhando a tendência nacional 18 , houve no município o fechamento de um hospital e redução de leitos psiquiátricos do Sistema Único de Saúde (SUS). Entre 2001 e 2002, são implantados sete CAPS, incluindo o de álcool e drogas (CAPSAD). Tais eventos levaram a uma importante mudança nas taxas de internação psiquiátrica - de 801 internações por 100 mil habitantes em 2001 para uma média de 491 internações por 100 mil nos anos de 2002 a 2006 (Departamento de Informática do SUS. Internações hospitalares do SUS - psiquiatria - Pelotas, 2001-2006. http:// tabnet.datasus.gov.br/cgi/tabcgi.exe?shi/cnv/ rxrs.def, acessado em 07/Ago/2008). Atualmen- te, com 350 mil habitantes e sete CAPS, o município é um dos que lideram o ranking nacional com cobertura de uma unidade para cada $50 \mathrm{mil}$ habitantes.

Considerando o cenário local no contexto das políticas em saúde mental, realizou-se uma avaliação em 2006/2007, com o apoio do Ministério da Saúde e do Conselho Nacional de Desenvolvimento Científico e Tecnológico (CNPq). O objetivo deste artigo é apresentar resultados selecionados do estudo, analisando a efetividade dos CAPS no cuidado de pessoas com sofrimento psíquico. Com isso, espera-se contribuir com as necessárias "correções de trajetória" com o objetivo de consolidar o modelo de atenção psicossocial no enfrentamento desses agravos no âmbito do SUS.

\section{Métodos}

O estudo foi do tipo coorte prospectiva, com um Estudo de Linha de Base (ELB - visita 1) e um acompanhamento (visita 2). Os indivíduos foram identificados e incluídos na coorte por seus prontuários nos CAPS, pelos quais foi obtido o endereço da residência. A primeira visita ocorreu de julho a outubro de 2006, e o acompanhamento ocorreu de maio a agosto de 2007. Os dados foram coletados em entrevistas domiciliares, por uma equipe de acadêmicos de Psicologia, Serviço Social (Universidade Católica de Pelotas - UCPel) e Enfermagem (Universidade Federal de Pelotas - UFPel), selecionados e capacitados para esse fim, após estudo piloto.

Um questionário foi respondido pelo usuário e outro por seu familiar responsável, após conhecimento do estudo e concordância em participar, mediante assinatura de termos de consentimento livre e esclarecido, tanto na visita 1 quanto na visita 2 .

Após codificação, tabulação e revisão, duas digitações independentes dos instrumentos foram realizadas no Epi Info 6.04 (Centers for Disease Control and Prevention, Atlanta, Estados Unidos), cuja estrutura foi preparada para verificação de amplitude e consistência. Após a edição final do banco de dados, ele foi convertido para o pacote estatístico SPSS 13.0 (SPSS Inc. Chicago, Estados Unidos), no qual foram feitas as análises.

O diagnóstico (segundo a 10ạ edição da Classificação Internacional de Doenças - CID-10 19), o tempo de freqüência ao CAPS e a modalidade de atendimento foram obtidos dos prontuários; as informações sobre internações hospitalares foram coletadas com os familiares. As demais informações utilizadas nas análises foram for- 
necidas diretamente pelos usuários dos CAPS. Todas as informações e instrumentos utilizados na coleta dos dados estão disponíveis na página eletrônica do estudo (http://www.capspelotas. com.br).

Foram considerados como exposição terapêutica: o uso de medicamentos nos 15 dias anteriores à entrevista e a participação em oficinas e grupos nos CAPS nos últimos 30 dias.

Para indicar a situação de saúde, utilizou-se a ocorrência de crise no último ano (linha de base) e no período entre as visitas (visita 2) e a hospitalização nos mesmos períodos, em hospital psiquiátrico ou geral.

Suas prevalências foram comparadas e estratificadas para a modalidade do atendimento no serviço - intensivo, semi-intensivo e não intensivo e para o tempo de freqüência ao serviço - menos de 1 ano; de 1-2,9 anos e 3 anos ou mais. O teste do qui-quadrado foi utilizado para a verificação das diferenças entre as proporções, considerando-se o nível de significância estatística de $5 \%$.

A pesquisa foi conduzida dentro dos padrões exigidos pela Declaração de Helsinki e aprovada pelo Comitê de Ética em Pesquisa da UCPel.

\section{Resultados}

\section{Descrição da amostra}

Entre os meses de abril e maio de 2006, foram identificados, por intermédio dos prontuários dos CAPS, 1.151 usuários, dos quais 1.013 foram entrevistados (88\%) na linha de base e 875 (76\%) na visita de acompanhamento. O percentual médio de perdas de $12 \%$ para a primeira visita deveu-se especialmente a endereços não localizados (7\%) e recusas (5\%). Foram registrados 16 óbitos entre as duas visitas domiciliares.

A Tabela 1 apresenta a distribuição dos usuários de acordo com características sóciodemográficas e o tipo de transtorno, de acordo com a modalidade do atendimento e o tempo de freqüência aos CAPS. A maioria dos freqüentadores dos CAPS era do sexo feminino (61\%). A média de idade foi de $45,6( \pm 12,5)$ anos e $75 \%$ tinham entre 30-59 anos; um terço tinha menos de 4 anos completos de escolaridade e $24 \%$ possuíam o primeiro grau completo. Pouco menos da metade (46\%) era casado(a) ou vivia com companheiro(a). Os transtornos de humor representaram a maior proporção dos diagnósticos (39\%), seguidos da esquizofrenia (24\%) e das neuroses (13\%). De acordo com a modalidade de tratamento, predominaram os pacientes não intensivos (46\%), seguidos dos semi-intensivos
(37\%) e dos intensivos (17\%). Em média, os usuários estavam freqüentando os CAPS há 3,5 anos $( \pm 1,9)$ e mais de metade $(58 \%)$ freqüentava há 3 anos ou mais (Tabela 1). Essas informações, utilizadas para as análises estratificadas, estavam disponíveis nos prontuários para, respectivamente, 919 e 928 usuários.

Os usuários com atendimento intensivo eram predominantemente homens e mais jovens, ao contrário dos não intensivos, com maior proporção de mulheres e com mais idade. Apesar de originalmente classificar os usuários para a emissão da Autorização de Procedimentos Ambulatoriais de Alta Complexidade/Custo, a modalidade de atendimento variou com os grupos diagnósticos: pessoas com transtornos de humor e neuroses eram mais freqüentes entre os não intensivos, enquanto aqueles com abuso de substâncias, esquizofrenia, retardo mental e transtornos orgânicos predominavam entre os semi-intensivos. $\mathrm{O}$ tempo de freqüência ao CAPS igualmente revelou diferenças, pois menores tempos de tratamento foram observados para pessoas com diagnóstico de abuso de substâncias e maiores tempos para diagnósticos de transtornos orgânicos e de personalidade.

\section{Características da exposição terapêutica}

Para todos os usuários, o uso de medicamentos foi reduzido de 93\% (IC95\%: 91-94) na visita 1 para 89\% (IC95\%: 86-91) na visita 2. Entretanto, reduções mais importantes no uso de psicofármacos foram observadas para os usuários não intensivos e para aqueles com maior tempo de freqüência ao CAPS (Tabela 2).

Em 2006, mais da metade dos usuários não participava de nenhuma oficina (61\%) ou grupo (60\%) no CAPS. Na visita de 2007, a participação em oficinas terapêuticas foi maior (44\%; IC95\%: 41-48) do que na visita 1 (39\%; IC95\%: 36-43). Também a participação nos diversos grupos aumentou de 40\% (IC95\%: 37-43) na visita 1 para 49\% (IC95\%: 45-52) no período. Significativamente maior participação em oficinas no interior dos CAPS foi observada para os usuários que freqüentavam os serviços há menos tempo e a participação em grupos foi significativamente maior entre os não intensivos e entre os que freqüentavam os serviços há 3 anos ou mais (Tabela 2). Ainda em relação à exposição terapêutica, uma parcela muito reduzida de usuários consultava com psiquiatra (5\%) ou psicólogo (2\%) fora do CAPS. 
Tabela 1

Distribuição dos usuários dos Centros de Atenção Psicossocial (CAPS) de acordo com características sócio-demográficas e de morbidade. Pelotas, Rio Grande do Sul, Brasil, 2006-2007.

\begin{tabular}{|c|c|c|c|c|c|c|c|}
\hline \multirow[t]{2}{*}{ Variável } & \multirow[t]{2}{*}{ N (\%) } & \multicolumn{3}{|c|}{ Modalidade de atendimento * } & \multicolumn{3}{|c|}{ Tempo de CAPS (anos) } \\
\hline & & $\begin{array}{l}\text { Intensivo } \\
\text { n (\%) }\end{array}$ & $\begin{array}{c}\text { Semi-intensivo } \\
\text { n (\%) }\end{array}$ & $\begin{array}{c}\text { Não intensivo } \\
n(\%)\end{array}$ & $\begin{array}{c}\text { Menos de } 1 \\
n(\%)\end{array}$ & $\begin{array}{l}1-2,9 \\
n(\%)\end{array}$ & $\begin{array}{c}3 \text { ou mais } \\
n(\%)\end{array}$ \\
\hline \multicolumn{8}{|l|}{ Sexo ** } \\
\hline Masculino & $395(39,0)$ & $84(23,0)$ & $136(37,0)$ & $145(40,0)$ & $32(8,6)$ & $137(37,0)$ & $204(55,0)$ \\
\hline Feminino & $618(61,0)$ & $69(13,0)$ & $207(37,0)$ & $278(50,0)$ & $35(6,3)$ & $185(33,0)$ & $335(60,0)$ \\
\hline \multicolumn{8}{|l|}{ 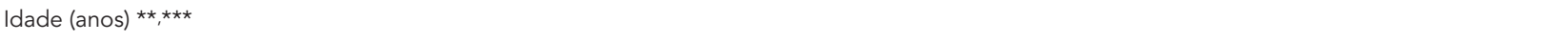 } \\
\hline $17-29$ & $127(12,0)$ & $27(23,0)$ & $41(35,0)$ & $48(41,0)$ & $15(13,0)$ & $32(28,0)$ & $66(58,0)$ \\
\hline $30-44$ & $331(33,0)$ & $63(21,0)$ & $122(40,0)$ & $117(39,0)$ & $26(8,0)$ & $126(41,0)$ & $156(51,0)$ \\
\hline $45-59$ & $426(42,0)$ & $53(14,0)$ & $146(37,0)$ & $191(49,0)$ & $21(5,0)$ & $126(32,0)$ & $244(62,0)$ \\
\hline 60 ou mais & $128(13,0)$ & $10(9,0)$ & $34(31,0)$ & $67(60,0)$ & $5(4,0)$ & $37(32,0)$ & $73(64,0)$ \\
\hline \multicolumn{8}{|l|}{ Escolaridade (anos) } \\
\hline Até 3 & $335(33,0)$ & $49(16,0)$ & $117(39,0)$ & $136(45,0)$ & $18(6,0)$ & $90(31,0)$ & $180(63,0)$ \\
\hline $4-7$ & $430(43,0)$ & $70(18,0)$ & $139(36,0)$ & $179(46,0)$ & $32(8,0)$ & $145(36,0)$ & $223(56,0)$ \\
\hline 8 ou mais & $239(24,0)$ & $33(15,0)$ & $83(38,0)$ & $104(47,0)$ & $17(7,0)$ & $84(36,0)$ & $131(57,0)$ \\
\hline \multicolumn{8}{|l|}{ Vive com companheiro(a) * } \\
\hline Sim & $463(46,0)$ & $52(13,0)$ & $154(37,0)$ & $207(50,0)$ & $26(6,0)$ & $148(34,0)$ & $256(60,0)$ \\
\hline Não & $549(54,0)$ & $101(20,0)$ & $188(37,0)$ & $216(43,0)$ & $41(8,0)$ & $174(35,0)$ & $283(57,0)$ \\
\hline \multicolumn{8}{|l|}{ Diagnóstico (CID-10) **,*** } \\
\hline Transtornos do humor & $452(39,0)$ & $42(11,0)$ & $128(34,0)$ & $203(54,0)$ & $19(5,0)$ & $122(32,0)$ & $240(63,0)$ \\
\hline Esquizofrenia & $271(24,0)$ & $65(29,0)$ & $84(38,0)$ & $74(33,0)$ & $15(7,0)$ & $74(34,0)$ & $129(59,0)$ \\
\hline Neuroses & $145(13,0)$ & $6(5,0)$ & $37(33,0)$ & $71(62,0)$ & $9(7,0)$ & $43(35,0)$ & $71(58,0)$ \\
\hline Retardo mental & $72(6,0)$ & $17(29,0)$ & $28(48,0)$ & $13(22,0)$ & $6(12,0)$ & $14(29,0)$ & $29(59,0)$ \\
\hline Abuso de substâncias & $65(6,0)$ & $4(8,0)$ & $35(70,0)$ & $11(22,0)$ & $5(10,0)$ & $40(80,0)$ & $5(10,0)$ \\
\hline Transtornos orgânicos & $50(4,0)$ & $9(22,0)$ & $19(46,0)$ & $13(32,0)$ & $1(2,0)$ & $10(23,0)$ & $32(74,0)$ \\
\hline Transtornos de personalidade & $20(2,0)$ & $7(37,0)$ & $3(16,0)$ & $9(47,0)$ & $0(0,0)$ & $3(18,0)$ & $14(82,0)$ \\
\hline Não especificado & $72(6,0)$ & $3(7,0)$ & $9(22,0)$ & $29(71,0)$ & $12(26,0)$ & $16(34,0)$ & $19(40,0)$ \\
\hline Total & $1.013(100,0)$ & $153(17,0)$ & $343(37,0)$ & $423(46,0)$ & $67(7,2)$ & $322(34,7)$ & $539(58,1)$ \\
\hline
\end{tabular}

* 8,2\% de informações ignoradas (dados obtidos em prontuários);

** $p<0,05$ para associação com modalidade de atendimento;

*** $p<0,05$ para associação com tempo de freqüência ao CAPS.

Impacto na situação de saúde dos usuários

Na visita $1,80 \%$ dos usuários relataram ter sofrido uma crise ou piora dos sintomas alguma vez na vida e $63 \%$ referiram crise ou piora nos 12 meses que antecederam a entrevista. O relato de crises caiu de 63\% (IC95\%: 60-67) na visita 1 para $30 \%$ (IC95\%: 26-34) na visita 2, em todas as categorias de modalidade e tempo de CAPS (Figura 1). Após freqüentar os CAPS, $24 \%$ dos usuários referiram ausência de crises, $60 \%$ tiveram com menor freqüência e $70 \%$ com menor intensidade.

As hospitalizações foram reduzidas de 16\% nos 12 meses anteriores à visita 1 (IC95\%: 14-19) para 12\% (IC95\%: 9-15) no período entre a visita
1 e a visita 2. Ao estratificar, observou-se redução significativa entre usuários em atendimento intensivo e aqueles com maior tempo de freqüência ao serviço.

Também foi possível constatar que, quanto maior o tempo de freqüência ao CAPS, maior o tempo decorrido desde a última internação psiquiátrica. Usuários que freqüentavam o serviço há menos de um ano estavam, em média, a 48 meses da última internação; os que estavam freqüentando entre um e 2,9 anos tiveram sua última internação há 66 meses e os que eram atendidos há 3 anos ou mais, o tempo médio foi de 88 meses. Esta tendência se manteve para pacientes com retardo mental, transtornos de humor e esquizofrenia. 
Comparação entre variáveis do plano terapêutico, de acordo com a modalidade de atendimento e o tempo de freqüência aos Centros de Atenção Psicossocial (CAPS). Pelotas, Rio Grande do Sul, Brasil, 2006-2007.

Uso de medicamentos

(\%)
Participação em grupos

(\%)

\begin{tabular}{|c|c|c|c|c|}
\hline \multicolumn{5}{|c|}{ Modalidade de atendimento } \\
\hline \multicolumn{5}{|l|}{ Intensivo } \\
\hline Visita 1 & 96,1 & 70,6 & 56,2 & 153 \\
\hline Visita 2 & 91,6 & 75,2 & 63,6 & 131 \\
\hline Valor de $\mathrm{p}$ & 0,113 & 0,346 & 0,221 & - \\
\hline \multicolumn{5}{|l|}{ Semi-intensivo } \\
\hline Visita 1 & 93,3 & 60,9 & 46,6 & 343 \\
\hline Visita 2 & 90,7 & 64,7 & 49,8 & 300 \\
\hline Valor de $\mathrm{p}$ & 0,219 & 0,329 & 0,445 & - \\
\hline \multicolumn{5}{|l|}{ Não intensivo } \\
\hline Visita 1 & 91,6 & 17,5 & 29,8 & 423 \\
\hline Visita 2 & 86,7 & 18,7 & 41,6 & 362 \\
\hline Valor de $p$ & 0,032 & 0,639 & 0,000 & - \\
\hline \multicolumn{5}{|c|}{ Tempo de freqüência ao CAPS (anos) } \\
\hline \multicolumn{5}{|l|}{ Menos de 1} \\
\hline Visita 1 & 82,1 & 38,8 & 20,9 & 67 \\
\hline Visita 2 & 73,7 & 62,5 & 28,1 & 57 \\
\hline Valor de $\mathrm{p}$ & 0,258 & 0,006 & 0,352 & - \\
\hline \multicolumn{5}{|l|}{$1-2,9$} \\
\hline Visita 1 & 90,7 & 41,9 & 38,5 & 322 \\
\hline Visita 2 & 86,0 & 42,0 & 42,0 & 278 \\
\hline Valor de $\mathrm{p}$ & 0,071 & 0,968 & 0,472 & - \\
\hline \multicolumn{5}{|l|}{3 ou mais } \\
\hline Visita 1 & 95,7 & 39,3 & 45,4 & 539 \\
\hline Visita 2 & 92,1 & 44,8 & 55,2 & 468 \\
\hline Valor de $p$ & 0,015 & 0,076 & 0,002 & - \\
\hline
\end{tabular}

\section{Discussão}

Na área da saúde mental e da reforma psiquiátrica, ainda são escassos estudos que avaliam a efetividade dos serviços substitutivos às instituições manicomiais. Acredita-se que o delineamento de coorte adotado nesta pesquisa é o mais adequado para a verificação de mudanças em desfechos individuais potencialmente atribuíveis a uma exposição. Neste caso, o interesse foi avaliar se usuários de CAPS teriam indicadores de sua situação de saúde positivamente associados à sua exposição terapêutica nos serviços, investigando-se este efeito de acordo com potenciais fatores intervenientes.

Apesar das perdas de acompanhamento, inerentes aos estudos de coorte, considera-se que a abrangência da amostra e a coleta de dados primários em entrevistas domiciliares são aspectos que diferenciam os achados descritos neste estudo daqueles oriundos de avaliações com base em dados secundários ou realizadas com delineamento transversal. Ainda do ponto de vista metodológico, análises estratificadas pelo tempo de freqüência ao CAPS e pela modalidade de atendimento foram necessárias, pois ambas as variáveis poderiam influenciar os desfechos avaliados, dada a sua potencial distribuição desigual entre os subgrupos diagnósticos.

De modo geral, a falta de informações para uma variável pode ser considerada um problema na análise dos dados. Neste estudo, para $6 \%$ dos usuários dos CAPS não foi possível conhecer o diagnóstico, o que revela que os serviços podem melhorar a qualidade dos seus registros nos prontuários. Em outros estudos, esta proporção foi ainda maior, de $9 \% 20$ e de $17 \% 21$. 
Figura 1

Ocorrência de crises de acordo com a modalidade e o tempo de freqüência ao Centro de Atenção Psicossocial (CAPS).

Pelotas, Rio Grande do Sul, Brasil, 2006-2007.

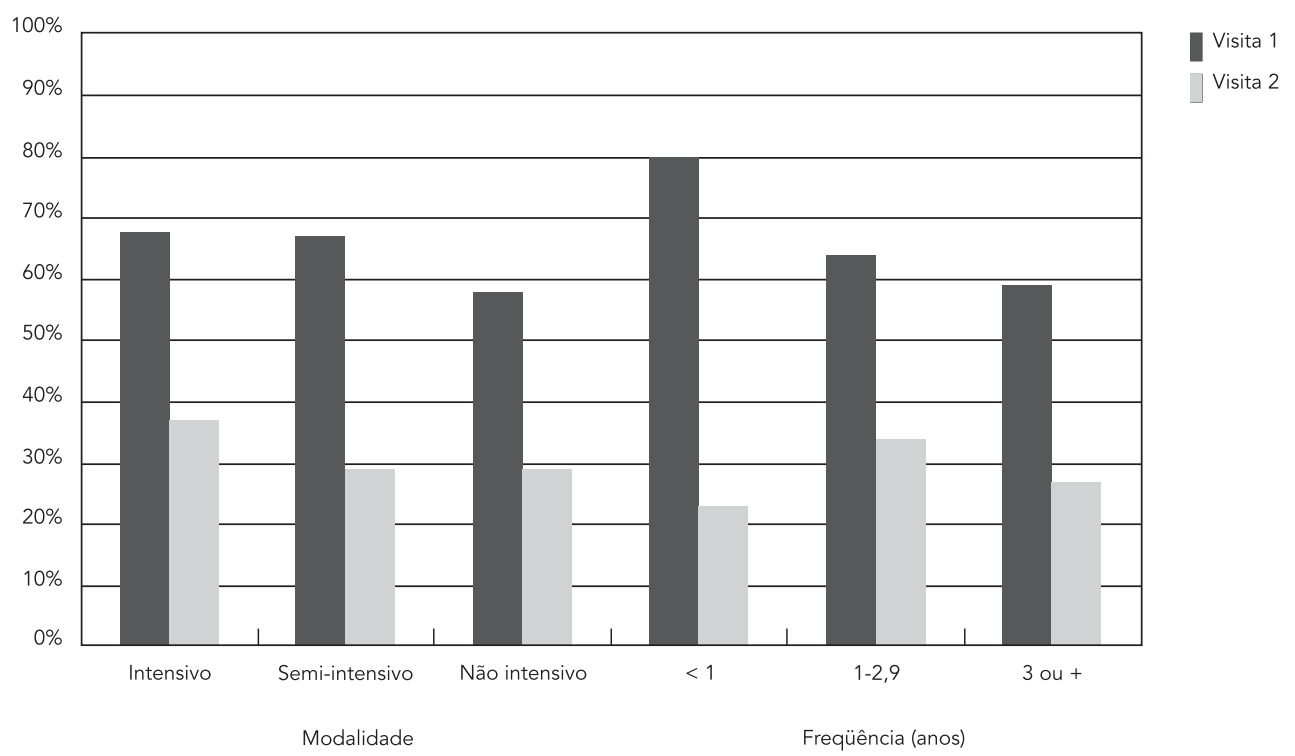

Nota: $p<0,001$

A distribuição por gênero, com leve predomínio de mulheres e do perfil de morbidade psíquica da amostra foram similares às descritas por Pelisoli \& Moreira 21, no Rio Grande do Sul, e por Terzian et al. 20 na Itália. Entretanto, os sujeitos deste estudo tinham mais idade do que os descritos por esses autores.

A utilização de terapêutica medicamentosa atingia quase $100 \%$ dos usuários estudados, mas sua redução deve ser interpretada com cautela. No período em que a pesquisa foi realizada, durante dois meses e meio, a gestão municipal teve problemas com a disponibilidade de medicamentos aos CAPS. Assim, o menor uso de medicamentos pode ser devido, em parte, a este desabastecimento, o que, frente aos demais indicadores, se configuraria como um reforço aos benefícios da estratégia dos cuidados. Tal suposição ganha força com o aumento da participação dos usuários em oficinas e grupos terapêuticos no mesmo período, sobretudo entre os não intensivos e os com menor tempo de CAPS. Além disso, a redução no uso de medicamentos foi significativa apenas para os usuários do regime não intensivo e com maior tempo de freqüência ao serviço, que eventualmente podem prescindir deste recurso terapêutico, se tiverem acesso a outras estratégias de cuidado.

Além de caracterizar o perfil dos freqüentadores dos CAPS de um município de médio porte, os dados sugerem que os CAPS estão cumprindo seu papel substitutivo aos hospitais psiquiátricos e, embora ainda com muitos problemas, revelam um efeito positivo do cuidado prestado pelos CAPS aos portadores de sofrimento psíquico. Este efeito pode ser observado na redução da ocorrência de crises para todos os usuários e das internações hospitalares para os intensivos e com maior tempo de freqüência ao serviço. É importante salientar que, com o maior tempo de inserção nos CAPS, os usuários tendem a diminuir a medicação, pois as diferenças foram significativas apenas no grupo com maior tempo de tratamento.

As internações hospitalares, atendendo diretrizes da política nacional de saúde mental 22 , vêm sendo gradativamente reduzidas. Em Pelotas, a redução nas internações observadas no período 2001 a 2006 (Departamento de Informática do SUS. Internações hospitalares do SUS psiquiatria - Pelotas, 2001-2006. http://tabnet. datasus.gov.br/cgi/tabcgi.exe?shi/cnv/rxrs.def, 
acessado em 07/Ago/2008) coincide com o fechamento de um hospital psiquiátrico e com o início da implantação dos CAPS em 2002.

Além disso, os dados do acompanhamento registraram quedas persistentes entre a visita 1 e a visita 2. Este resultado parece ser um forte indicador do efeito dos cuidados dispensados nos CAPS na redução das internações, uma vez que sua aferição foi realizada após a redução do número de leitos disponíveis pelo SUS no município. Como Pelotas é referência regional para hospitalizações psiquiátricas, não se acredita que pacientes locais busquem este serviço em outros municípios, pelo menos em proporções que possam afetar estes resultados.

Em relação às crises ou pioras, deve-se observar que a magnitude da diferença em sua ocorrência entre a primeira e a segunda visita, em que pese o menor período recordatório - 12 para 9 meses, foi bastante significativa, independente da modalidade do atendimento e do tempo de freqüência ao CAPS. Este é um dado baseado no relato dos familiares e, portanto, bastante confiável, dado que as crises, quando ocorrem, sempre envolvem a família.
Apesar das limitações inerentes a serviços como os CAPS, os usuários com sintomas mais severos (intensivos) tendem a diminuir suas intercorrências ao longo do tempo, fato que fala a favor da equidade do novo modelo de atenção, já que usuários de modalidade semi-intensiva e não intensiva não diminuíram significativamente a necessidade de internação hospitalar, ainda o indicador mais robusto para avaliar o papel dos serviços substitutivos.

A suposição de um efeito positivo da nova estratégia de cuidado é reforçada pelo tempo médio de freqüência dos usuários entrevistados aos serviços. Os vínculos estabelecidos com as equipes proporcionam aos técnicos a possibilidade de intervir rapidamente nos sinais de piora evitando seu progresso.

Assim, em que pese a necessidade de ampliação da qualidade dos CAPS, dos investimentos em infra-estrutura e de melhorias organizacionais, este estudo mostrou que a estratégia é efetiva e deve ser reforçada no âmbito do SUS. Novos desafios precisam ser enfrentados, como o fortalecimento de redes de apoio social e comunitário, a articulação com a rede básica de saúde e a extensão das políticas aos familiares, que se configuram como um importante pilar de sustentação do novo modelo.

\section{Resumo}

Objetivo foi analisar a efetividade dos Centros de Atenção Psicossocial (CAPS) no cuidado de portadores sofrimento psíquico em Pelotas, Rio Grande do Sul, Brasil. Coorte prospectiva, com usuários de todos os CAPS do município em duas visitas domiciliares. Três variáveis de exposição terapêtica e duas da situação de saúde foram comparadas e estratificadas pela modalidade de atendimento e pelo tempo de freqüência ao serviço. Foram entrevistados 1.013 usuários na visita 1 e 875 na visita 2. Os transtornos mais freqüentes foram os de humor (39\%), esquizofrenia (24\%) e neuroses (13\%). Mais de 50\% recebiam atendimento intensivo ou semi-intensivo e quase $60 \%$ freqüentavam o serviço há três anos ou mais. Houve uma redução significativa na ocorrência de crises ( $p=0,000)$, e o número de internações psiquiátricas foi menor entre aqueles em atendimento intensivo $(p=0,002)$ e com maior tempo de CAPS ( $p=0,003)$. Entre os não-intensivos, diminuiu o uso de medicamentos $(p=0,032)$ e aumentou a participação em grupos ( $p=0,000)$. Os CAPS podem ser considerados efetivos na assistência aos portadores de sofrimento psíquico.

Serviços de Saúde Mental; Avaliação de Serviços de Saúde; Estresse Psicológico 


\section{Colaboradores}

E. Tomasi, L. A. Facchini, R. X. Piccini e H. Gonçalves participaram da concepção e delineamento do estudo, análise e interpretação dos dados, revisão e aprovação da versão final. E. Thumé colaborou na concepção e delineamento do estudo, revisão e aprovação da versão final. R. A. Silva e S. M. Silva contribuíram na análise e interpretação dos dados, revisão e aprovação da versão final.

\section{Referências}

1. The WHO World Mental Health Survey Consortium. Prevalence, severity, and unmet need for treatment of mental disorders in the World Health Organization World Mental Health Surveys. JAMA 2004; 291:2581-90.

2. Andreoli SB, Almeida Filho N, Coutinho ESF, Mari JJ. Identificação de casos psiquiátricos em estudos epidemiológicos multifásicos: métodos, problemas e aplicabilidade. Rev Saúde Pública 2000; 34:475-83.

3. Dias-da-Costa JS, Menezes AMB, Olinto MTA, Gigante DP, Macedo S, Britto MAP, et al. Prevalência de distúrbios psiquiátricos menores na cidade de Pelotas, RS. Rev Bras Epidemiol 2002; 5:164-73.

4. Pádua AC, Gama CS, Lobato MIR, Abreu PSB. Esquizofrenia. In: Cordioli AV, organizador. Psicofármacos baseados em evidências. São Paulo: Editora Artmed; 2005. p. 343-9.
5. Shansis FM, Cordioli AV. Transtornos do espectro do humor bipolar. In: Cordioli AV, organizador. Psicofármacos baseados em evidências. São Paulo: Editora Artmed; 2005. p. 329-42.

6. Lima MS. Epidemiologia e impacto social. Rev Bras Psiquiatr 1999; 21 Suppl 1:Sl1-5.

7. Galduroz JCF, Noto AR, Nappo AS, Carlini ELA. First household survey on drug abuse in São Paulo, Brazil, 1999: principal findings. São Paulo Med J 2003; 121:231-7.

8. Dias-da-Costa JS, Silveira MF, Gazalle FK, Oliveira SS, Hallal PC, Menezes AMB, et al. Consumo abusivo de álcool e fatores associados: estudo de base populacional. Rev Saúde Pública 2004; 38:284-91.

9. Xavier FMF, Ferraz MPT, Trenti CM, Argimon I, Bertollucci PH, Poyares D, et al. Transtorno de ansiedade generalizada em idosos com 80 anos ou mais. Rev Saúde Pública 2001; 35:294-302. 
10. Amarante P. Novos sujeitos, novos direitos: o debate em torno da reforma psiquiátrica. Cad Saúde Pública 1995; 11:491-4.

11. Alverga AR, Dimenstein M. A reforma psiquiátrica e os desafios na desinstitucionalização da loucura. Interface Comun Saúde Educ 2006; 10:299-316.

12. Brasil. Lei no. 10.216, de 6 de abril de 2001, dispõe sobre a proteção e os direitos das pessoas portadoras de transtornos mentais e redireciona o modelo assistencial em saúde mental. Diário Oficial da União 2001; 9 abr

13. Ministério da Saúde. Saúde mental no SUS: os Centros de Atenção Psicossocial. Brasília: Ministério da Saúde; 2004.

14. Borges CF, Baptista TWF. O modelo assistencial em saúde mental no Brasil: a trajetória da construção política de 1990 a 2004. Cad Saúde Pública 2008; 24:456-68.

15. Secretaria de Atenção à Saúde, Ministério da Saúde. Saúde mental em dados. Brasília: Ministério da Saúde; 2007.

16. Habicht JP, Victora CG, Vaughan JP. Evaluation designs for adequacy, plausibility and probability of public health programme performance and impact. Int J Epidemiol 1999; 28:10-8.
17. Tomasi E. Os CAPS e os cuidados psicossociais: cenários e possibilidades na evolução dos portadores de sofrimento psíquico em Pelotas, RS. Relatório final. Pelotas: Universidade Católica de Pelotas; 2008.

18. Ministério da Saúde. Portaria no ${ }^{\circ} .1174 / \mathrm{GM}$ de 7 de julho de 2005. Diário Oficial da União 2005; 8 jul.

19. Organização Mundial da Saúde. Classificação estatística internacional de doenças e problemas relacionados à saúde, 10a revisão. v. 1. São Paulo: Centro Colaborador da OMS para a Classificação de Doenças em Português; 1995.

20. Terzian E, Sternai E, Barbato A, Tognoni G, Saraceno B. Epidemiology of psychiatric care of patients with severe mental disorders in Italy. Rationale and design of a prospective study, and characteristics of the cohort. Italian Collaborative Study Group. Soc Psychiatry Psychiatr Epidemiol 1997; 32:298302.

21. Pelisoli CL, Moreira AK. Caracterização epidemiológica dos usuários do Centro de Atenção Psicossocial Casa Aberta. Rev Psiquiatr Rio Gd Sul 2005; 27:270-7.

22. Coordenação Geral de Saúde Mental, Secretaria de Atenção à Saúde, Ministério da Saúde. Reforma psiquiátrica e política de saúde mental no Brasil. Brasília: Ministério da Saúde; 2005.

Recebido em 07/Mar/2009

Versão final reapresentada em 22/Out/2009

Aprovado em 18/Dez/2009 